\title{
Adaptive Fuzzy Control of Uncertain Robotic Manipulator
}

\author{
Jinglei Zhou ${ }^{1}$ and Qunli Zhang $\mathbb{D}^{2}$ \\ ${ }^{1}$ College of Machine and Electrical Engineering, Heze University, Heze, Shandong 274015, China \\ ${ }^{2}$ College of Mathematics and Statistics, Heze University, Heze, Shandong 274015, China \\ Correspondence should be addressed to Qunli Zhang; qunli-zhang@126.com
}

Received 27 December 2017; Revised 13 February 2018; Accepted 8 March 2018; Published 6 May 2018

Academic Editor: Qingling Zhang

Copyright @ 2018 Jinglei Zhou and Qunli Zhang. This is an open access article distributed under the Creative Commons Attribution License, which permits unrestricted use, distribution, and reproduction in any medium, provided the original work is properly cited.

\begin{abstract}
This paper designs a kind of adaptive fuzzy controller for robotic manipulator considering external disturbances and modeling errors. First, $n$-link uncertain robotic manipulator dynamics based on the Lagrange equation is changed into a two-order multipleinput multiple-output (MIMO) system via feedback technique. Then, an adaptive fuzzy logic control scheme is studied by using sliding theory, which adopts the adaptive fuzzy logic systems to estimate the uncertainties and employs a filtered error to make up for the approximation errors, hence enhancing the robust performance of robotic manipulator system uncertainties. It is proved that the tracking errors converge into zero asymptotically by using Lyapunov stability theory. Last, we take a two-link rigid robotic manipulator as an example and give its simulations. Compared with the existing results in the literature, the proposed controller shows higher precision and stronger robustness.
\end{abstract}

\section{Introduction}

The robotic control system is a kind of strongly coupling and highly nonlinear dynamical system. One of the main control aims is to guarantee the factual motion traces to track the given traces under the ideal dynamic quality. When the precise robotic mathematic model is established, this aim is achieved easily. However, at the practical operation scene, the robotic control systems is always influenced by the external stochastic disturbances, including the Coulomb force and the Friction force, and the inside parameter perturbations. Therefore, the mathematic model established under the face of ideal instance cannot work well and finding the robust controller which can compensate the uncertainties of practical mathematic model is very important [1-5].

With the development of cybernetics, more and more intelligent control methods such as neural control and fuzzy control are applied into some nonlinear dynamic systems. Among these intelligent control methods, the fuzzy logic is used more widely because of its nonlinear approximation ability. In [6], a fuzzy terminal sliding mode controller is developed for linear systems with mismatched timevarying uncertainties. In [7], an adaptive fuzzy tracking control is suggested for a class of MIMO nonlinear systems considering the system uncertainties, unknown nonsymmetric input saturation, and external disturbances. In [8], an adaptive fuzzy controller based on any observer for a class of affine nonlinear system is developed. And the performance of the developed controller is demonstrated in an inverted pendulum system and a chaotic system. In [9], a new adaptive fuzzy terminal sliding mode tracking controller is presented for a class of nonlinear systems. This control strategy is proved to achieve favourable control performance considering parameter variations and external disturbances. In [10], a fuzzy mixed $H_{2} / H_{\infty}$ sampled-data control scheme is proposed for nonlinear systems, and the effectiveness and feasibility of the proposed controller are illustrated by using a computer simulated truck-trailer system. In [11], an adaptive backstepping fuzzy controller for servo systems with unknown parameters and nonlinear backlash is developed. The developed controller shows that it has higher accuracy and robustness in performances than PID controller. In [12], a robust adaptive controller using a fuzzy compensator for MEMS triaxial gyroscope with nonlinearities, model uncertainties, and external disturbances is proposed. In [13], a robust adaptive fuzzy compensation based tracking controller including a second-order filler with physical constraints is proposed for nonlinear ship 
course-keeping system with modeling errors and external disturbances.

Of course, the application of the fuzzy control strategy in robotic manipulator systems' tracking problem has been a hot field. In [14], a robust fuzzy controller is designed for robotic manipulator to ensure both global stability and robust performance. In [15], a backstepping adaptive fuzzy control strategy is suggested to ensure the tracking errors convergence into zero. However, both $[14,15]$ do not consider the effect of the approximation error. In [16], the approximation error is considered, but the proposed adaptive fuzzy backstepping controller can only achieve uniform ultimate boundness, rather than asymptotical stability. In addition, these references make little difference between modeling uncertainty and external disturbance or neglect the external disturbance. In fact, there is no relationship between external disturbances and system parameters. Moreover, it is difficult to guarantee the stability, error convergence, and robustness based on offline trained fuzzy logic because of the highly nonlinear of the fuzzy logic.

In this paper, an adaptive fuzzy logic based feedback control scheme is developed by using the sliding theory $[17,18]$ to enhance the robustness of the closed-loop system. The adaptive fuzzy logic controller consists of the fuzzy logic approximation module and the sliding mode control module. The first module is used to model the dynamics of nonlinear systems and the second module is adopted to compensate for the approximation errors such that the presented control scheme can guarantee the asymptotic convergence of the trajectory tracking error and the global stability of the closedloop system. The main contribution of this scheme can be summarized as follows:

(1) This paper adopts feedback control technique to transform the Lagrange equation into a concise state equation, which can be studied by adopting linear control technology. Compared with the nonlinear equations, there are a lot of achievements regarding linear equation: optimal control [19], fuzzy cerebellar model articulation control [20], and so on [2,21].

(2) External disturbances and uncertain modeling errors are considered meanwhile for developing the weight updating algorithms which are updated online by employing Lyapunov stability theory. The Lyapunov stability theory is also employed to prove that the designed controller guarantees the stability of tracking.

(3) The two lumped modeling uncertainties are adopted by fuzzy logic system to reduce the number of fuzzy rules hereinbelow.

The organization of the paper is as follows. After a general description of the uncertain robotic system, the dynamical model based on the Lagrange equation is transformed into a more concise state equation via feedback control technique in Section 2. In Section 3, a lot of space is used to develop a kind of new controller that is adaptive fuzzy logic. The example simulations for a two-link manipulator are given in Section 4 , and the paper has been concluded in Section 5.

\section{Description of Robotic System}

The $n$-link robotic manipulator dynamical model based on the Lagrange equation can be described by the following twoorder differential equation [22]:

$$
M(q) \ddot{q}+h(q, \dot{q})=\tau+\tau_{d},
$$

where $q, \dot{q}, \ddot{q} \in R^{n}$ are the joint displacement, the joint velocity, and the joint acceleration; $M(q) \in R^{n \times n}$ is the inertia matrix of the robot; $h(q, \dot{q}) \in R^{n} d$ is the coupled vector by the Coriolis, centrifugal, and gravitational force; $\tau \in R^{n} r$ is the generalized control force; $\tau_{d} \in R^{n}$ stands for the external uncertain disturbance, which is bounded. The robotic model has the following property.

Property 1. $M(q) i$ is symmetric and positive definite. For all $q$ it is bounded; that is, there are two positive numbers $\lambda_{m} \leq$ $\lambda_{M}$ satisfying the following inequality:

$$
\lambda_{m} \leq\|M(q)\| \leq \lambda_{M} .
$$

Besides external disturbances, the modeling uncertainties will be considered also in the actual robotic manipulator system. Then, (1) is rewritten as

$$
M_{0}(q) \ddot{q}+h_{0}(q, \dot{q})=\tau+\tau_{d}+\rho,
$$

where $\rho=-\Delta M(q) \ddot{q}-\Delta h(q, \dot{q})$ is the lumped modeling error, $\Delta M(q)$ and $\Delta h(q, \dot{q})$ are unknown parts, and $M_{0}(q)$ and $h_{0}(q, \dot{q})$ are known parts.

Select the following generalized control force:

$$
\tau=h_{0}(q, \dot{q})+M_{0}(q) u .
$$

From (3) (4), the following dynamical equation is obtained:

$$
\ddot{q}=u+M_{0}^{-1}(q) \tau_{d}+M_{0}^{-1}(q) \rho .
$$

Let $d=M_{0}^{-1}(q) \tau_{d}$ and $f(q, \dot{q}, \ddot{q})=M_{0}^{-1}(q) \rho$; then, the more concise state equation is obtained as follows:

$$
\ddot{q}=f(q, \dot{q}, \ddot{q})+u+d .
$$

Then, the control aim of system (6) is to design $u$ to make the actual trace follow the given $q_{d}$ asymptotically. Here the given $q_{d}$ is a two-order continuously bounded differentiable function.

Remark 2. Because of Property 1 and the boundness of $\tau_{d}, d=M_{0}^{-1}(q) \tau_{d}$ is also bounded; that is to say, there is

$$
\left|d_{i}\right| \leq d_{0}
$$

where $d_{0}$ is a positive constant number.

\section{Adaptive Fuzzy Controller}

3.1. Nominal Model. When there is not modeling uncertainty and external uncertain disturbance $\tau_{d}$ equals zero, system (1) is called the nominal model [22] of robotic manipulator. Then 
system (6) becomes $\ddot{q}=u$. There exists a linear feedback control law:

$$
u=\ddot{q}_{d}-\alpha \dot{e}-\beta e,
$$

which makes the dynamics of system (6) become

$$
\ddot{e}+\alpha \dot{e}+\beta e=0,
$$

where $e=q-q_{d}$ is the trajectory tracking error and $\alpha, \beta \in$ $R^{n \times n}$ are positive definite matrices. They are also usually specified to be diagonal matrices for decoupling.

Obviously, (9) is stable; that is, the tracking error $e$ asymptotically converges to zero. In fact, (9) can be rewritten as

$$
\dot{S}=A S,
$$

where

$$
\begin{aligned}
& S=\left[\begin{array}{l}
e \\
\dot{e}
\end{array}\right], \\
& A=\left[\begin{array}{cc}
0 & I \\
-\beta & -\alpha
\end{array}\right] .
\end{aligned}
$$

And $A$ is a positive definite matrix [23]. Hence, there exists a positive definite matrix $P$ meeting the following Lyapunov equation:

$$
P A+A^{\mathrm{T}} P=-Q,
$$

where $Q$ is a positive definite matrix too.

3.2. Uncertainty Exists. In actual system of robotic manipulator, there are always uncertainties including modeling errors and external disturbances. That is to say, $f(q, \dot{q}, \ddot{q})$ exits and $d$ does not equal zero in (6).

In order to still use the feedback control technique, the uncertainty $f(q, \dot{q}, \ddot{q})$ needs to be estimated. Let's say the estimation is $\widehat{f}(q, \dot{q}, \ddot{q})$. And then, in a relatively straightforward manner, we can get the following modified control law:

$$
u=u_{a l}=-\hat{f}(q, \dot{q}, \ddot{q})+\ddot{q}_{d}-\alpha \dot{e}-\beta e .
$$

Substituting (13) into (6), the following error dynamics will emerge:

$$
\ddot{e}+\alpha \dot{e}+\beta e=(f(q, \dot{q}, \ddot{q})-\widehat{f}(q, \dot{q}, \ddot{q}))+d .
$$

Due to $f(q, \dot{q}, \ddot{q})-\widehat{f}(q, \dot{q}, \ddot{q}) \neq 0$ and $d \neq 0$, the stability of error equation (14) cannot be guaranteed easily.

Therefore, in order to eliminate the effects of approximation error and outside disturbance $d$, we need to redesign controller (13) by adding a compensation term $u_{s l}$; that is, the control input $u$ has the following expression:

$$
u=u_{a l}+u_{s l} \text {. }
$$

Since it is proved that fuzzy logic can approximate a large range of nonlinear system to any given degree of accuracy, in this paper the estimation $\widehat{f}(q, \dot{q}, \ddot{q})$ can be gotten by using the fuzzy logic $[16,24,25]$.

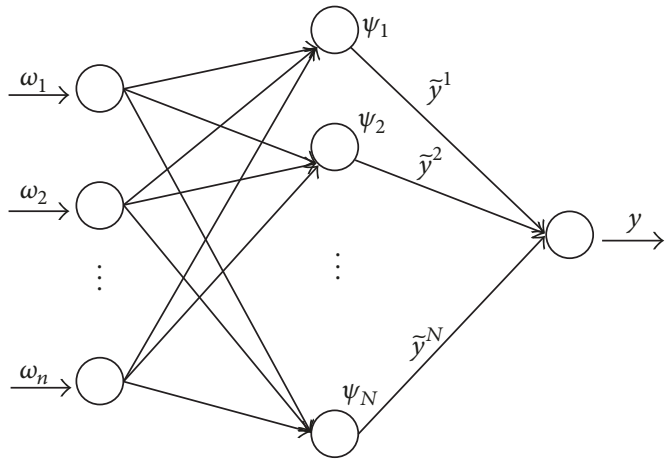

FIGURE 1: Structure of a fuzzy logic system.

3.3. Fuzzy Logic System. A fuzzy logic system contains four parts [24], which are the knowledge base, the fuzzifier, the fuzzy inference engine working on fuzzy rules, and the defuzzifier, respectively. The primal fuzzy system is fixed and uniformity. To hold the consistent performance of the fuzzy system under this circumstance where there is a lot of uncertainty or unknown development tendency in system parameters and structures, the fuzzy system should possess adaptive performance.

Without loss of generality, the output of an uncertain system can be assumed as $f(\omega)$. The fuzzy inference engine adopts the fuzzy if-then rules to form a mapping from an input vector $\omega=\left[\omega_{1}, \ldots, \omega_{n}\right]^{T} \in R^{n}$ to an output variable $y \in R$. The $i$ th fuzzy rule is of the form

$$
R^{i} \text { : If } \omega_{1} \text { is } \mu_{1}^{i} \text { and } \ldots \omega_{n} \text { is } \mu_{n}^{i} \text {, then } y \text { is } B^{i} \text {, }
$$

where $\mu_{1}^{i}, \ldots, \mu_{n}^{i}$ and $B^{i}$ are fuzzy sets characterized by fuzzy membership functions, for example, the Gaussian type.

Take the singleton fuzzifier, the product inference engine, and the center-average defuzzifier; then, the output variable $y$ can be described as

$$
y=\frac{\sum_{i=1}^{N} \tilde{y}^{i} \prod_{j=1}^{n} \mu_{j}^{i}\left(\omega_{j}\right)}{\sum_{i=1}^{N}\left(\prod_{j=1}^{n} \mu_{j}^{i}\left(\omega_{j}\right)\right)}=\theta^{\mathrm{T}} \psi(\omega),
$$

where $N$ is the number of fuzzy rules, $\theta=\left[\tilde{y}^{1}, \ldots, \tilde{y}^{N}\right]^{\mathrm{T}}$ with $\tilde{y}^{i}$ being an adjustable value where the fuzzy membership function $\mu_{B^{i}}\left(\widetilde{y}^{i}\right)$ has the maximum value, choosing $\mu_{B^{i}}\left(\widetilde{y}^{i}\right)=$ 1 usually, and $\psi(\omega)=\left[\psi_{1}, \ldots, \psi_{N}\right]^{\mathrm{T}}$ is a fuzzy basis vector, whose element is of the form

$$
\psi_{l}=\frac{\prod_{j=1}^{n} \mu_{j}^{i}\left(\omega_{j}\right)}{\sum_{i=1}^{N}\left(\prod_{j=1}^{n} \mu_{j}^{i}\left(\omega_{j}\right)\right)}, \quad l=1, \ldots, N .
$$

And the adaptive fuzzy system can be considered as the type of a neural network, which is shown in Figure 1 [24-26].

3.4. Fuzzy Approximators. In this subsection we propose the use of fuzzy system to estimate the unknown function $f(q, \dot{q}, \ddot{q})$. Then, the approximation is sued to develop a welldefined adaptive controller with its adaptation law in order to satisfy control objective. 
First, for reducing the number of fuzzy rules hereinbelow, the uncertainty $f(q, \dot{q}, \ddot{q})$ can be represented as an addition of two functions:

$$
f(q, \dot{q}, \ddot{q})=f^{1}(q, \dot{q})+f^{2}(q, \ddot{q}),
$$

where

$$
\begin{aligned}
& f^{1}(q, \dot{q})=-M_{0}^{-1}(q) \Delta h(q, \dot{q}), \\
& f^{2}(q, \ddot{q})=-M_{0}^{-1}(q) \Delta M(q) \ddot{q} .
\end{aligned}
$$

And each function will be replaced by the fuzzy logic system $\hat{f}^{1}\left(q, \dot{q} \mid \theta^{1}\right)$ and $\hat{f}^{2}\left(q, \ddot{q} \mid \theta^{2}\right)$, respectively, which are of the form of (16) and (17):

$$
\begin{aligned}
\widehat{f}^{1}\left(q, \dot{q} \mid \theta^{1}\right) & =\left[\theta_{1}^{1 \mathrm{~T}} F^{1}(q, \dot{q}), \ldots, \theta_{n}^{1 \mathrm{~T}} F^{1}(q, \dot{q})\right]^{\mathrm{T}} \\
& =\theta^{1 \mathrm{~T}} F^{1}(q, \dot{q}) \\
\widehat{f}^{2}\left(q, \ddot{q} \mid \theta^{2}\right) & =\left[\theta_{1}^{2 \mathrm{~T}} F^{2}(q, \ddot{q}), \ldots, \theta_{n}^{2 \mathrm{~T}} F^{2}(q, \ddot{q})\right]^{\mathrm{T}} \\
& =\theta^{2 \mathrm{~T}} F^{2}(q, \ddot{q}) .
\end{aligned}
$$

Thus, there is

$$
\widehat{f}(q, \dot{q}, \ddot{q})=\theta^{1 \mathrm{~T}} F^{1}(q, \dot{q})+\theta^{2 \mathrm{~T}} F^{2}(q, \ddot{q}) .
$$

Letting the optimal parameter matrices of the fuzzy logic system be $\theta^{1 *}$ and $\theta^{2 *}$, respectively, the minimum approximation error vectors can be defined as follows:

$$
\begin{aligned}
& \varepsilon^{1}=f^{1}(q, \dot{q})-\theta^{1 * \mathrm{~T}} F^{1}(q, \dot{q}) \\
& \varepsilon^{2}=f^{2}(q, \ddot{q})-\theta^{2 * \mathrm{~T}} F^{2}(q, \ddot{q}) .
\end{aligned}
$$

And they are bounded according to the approximation principle of the fuzzy logic; that is to say, there are very small positive constants $\sigma^{1}$ and $\sigma^{2}$ satisfying

$$
\begin{aligned}
& \sigma^{1} \geq\left|\varepsilon_{i}^{1}\right|, \\
& \sigma^{2} \geq\left|\varepsilon_{i}^{2}\right|,
\end{aligned}
$$

where $\varepsilon_{i}^{1}$ and $\varepsilon_{i}^{2}$ denote the $i$ th element of the vector $\varepsilon^{1}$ and $\varepsilon^{2}$.

Define the approximation error of weight matrices as

$$
\begin{aligned}
& \widetilde{\theta}^{1}=\theta^{1}-\theta^{1 *}, \\
& \widetilde{\theta}^{2}=\theta^{2}-\theta^{2 *} .
\end{aligned}
$$

\subsection{Two Lemmas}

Lemma 3. If $a, c \in R^{n}$ are row vectors, $D \in R^{n \times n}$ is a square matrix; then there is

$$
a^{\mathrm{T}} D c=\operatorname{tr}\left(D^{\mathrm{T}} a c^{\mathrm{T}}\right) .
$$

Proof. Let a $=\left[\begin{array}{lll}a_{1} & \cdots & a_{n}\end{array}\right]^{\mathrm{T}}, c=\left[\begin{array}{lll}c_{1} & \cdots & c_{n}\end{array}\right]^{\mathrm{T}}$, and

$$
D=\left[\begin{array}{ccc}
d_{11} & \cdots & d_{1 n} \\
\vdots & \ddots & \vdots \\
d_{n 1} & \cdots & d_{n n}
\end{array}\right] .
$$

On the left,

$$
a^{\mathrm{T}} D c=\left[\sum_{i=1}^{n} a_{i} d_{i 1} \cdots \sum_{i=1}^{n} a_{i} d_{i 1 n}\right] c=\sum_{j=1}^{j-n} \sum_{i=1}^{n} a_{i} d_{i j} c_{j} .
$$

On the right, first

$$
a c^{\mathrm{T}}=\left[\begin{array}{ccc}
a_{1} c_{1} & \cdots & a_{1} c_{n} \\
\vdots & \ddots & \vdots \\
a_{n} c_{1} & \cdots & a_{n} c_{n}
\end{array}\right] .
$$

Then

$$
\begin{aligned}
D^{\mathrm{T}} a c^{\mathrm{T}} & =\left[\begin{array}{ccc}
d_{11} & \cdots & d_{n 1} \\
\vdots & \ddots & \vdots \\
d_{1 n} & \cdots & d_{n n}
\end{array}\right]\left[\begin{array}{ccc}
a_{1} c_{1} & \cdots & a_{1} c_{n} \\
\vdots & \ddots & \vdots \\
a_{n} c_{1} & \cdots & a_{n} c_{n}
\end{array}\right] \\
& =\left[\begin{array}{ccc}
\sum_{i=1}^{n} d_{i 1} a_{i} c_{1} & \cdots & \sum_{i=1}^{n} d_{i 1} a_{i} c_{n} \\
\vdots & \ddots & \vdots \\
\sum_{i=1}^{n} d_{i n} a_{i} c_{1} & \cdots & \sum_{i=1}^{n} d_{i n} a_{i} c_{n}
\end{array}\right],
\end{aligned}
$$

so

$$
\operatorname{tr}\left(D^{\mathrm{T}} a c^{\mathrm{T}}\right)=\sum_{j=1}^{j=n} \sum_{i=1}^{n} a_{i} d_{i j} c_{j}
$$

is equal to the left.

According to Barbalat's lemma [22], If one function $f(t)$ is uniformly continuous on $[0,+\infty)$ and its general integral exists, then $\lim _{t \rightarrow \infty} f(t)=0$.

3.6. Main Result. In order to eliminate the effects of the approximation error and the external disturbance, we design the second part $u_{s l}$ in (15) as follows:

$$
u_{s l}=-k_{s l} \operatorname{sgn}(\delta),
$$

where $\delta=\left[\begin{array}{ll}P_{21} & P_{22}\end{array}\right] S$ is the defined filtered error. $P_{21}$ and $P_{22}$ come from the solution $P$ of the Lyapunov equation (12), and the positive definite solution $P$ has the following form:

$$
P=\left[\begin{array}{ll}
P_{11} & P_{21} \\
P_{21} & P_{22}
\end{array}\right] .
$$




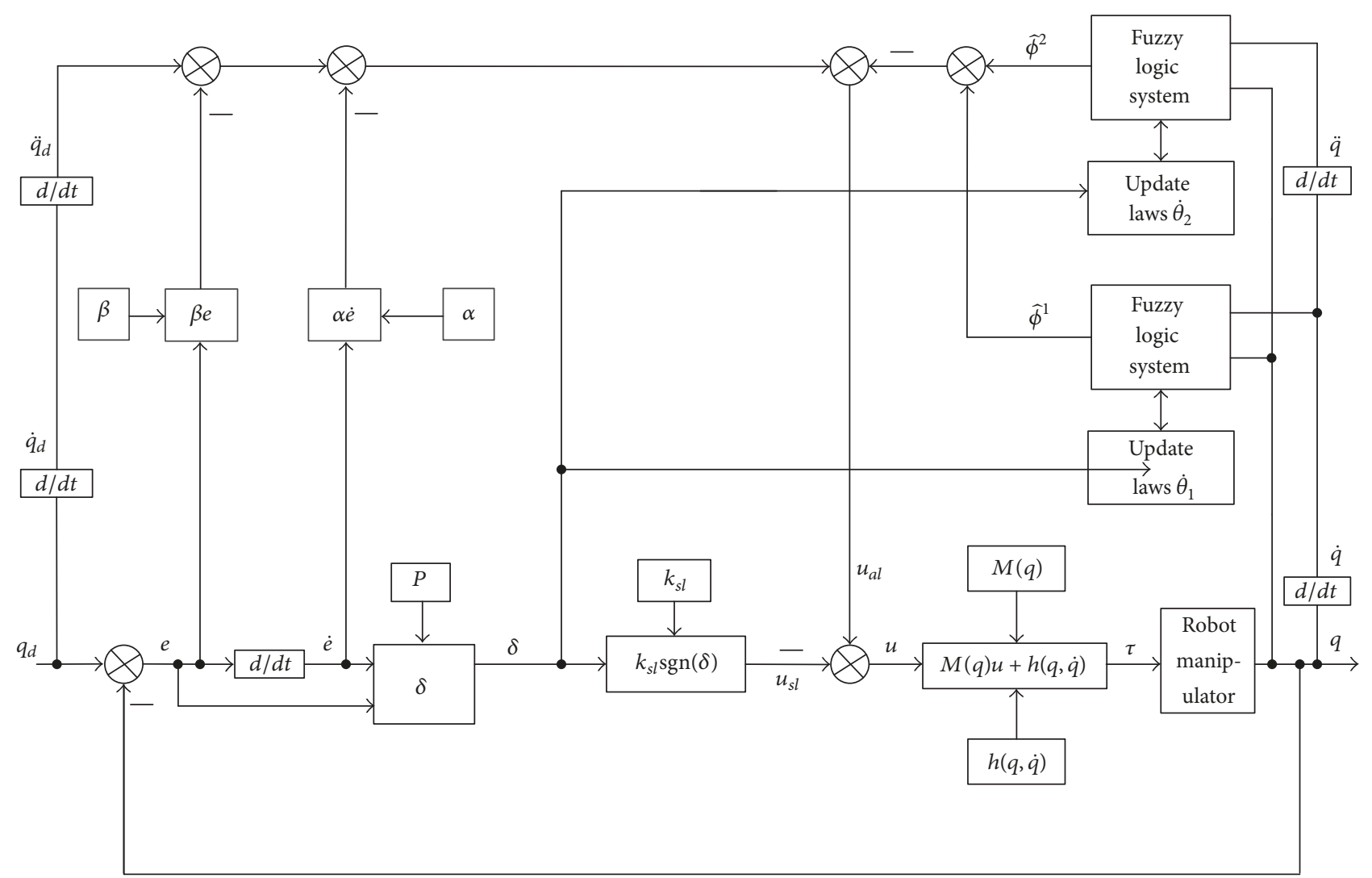

FIGURE 2: Architecture of the control scheme.

And $k_{s l}$ is the control gain given by the following expression:

$$
k_{s l}=\left(\sigma^{1}+\sigma^{2}+d_{0}\right) I_{n},
$$

where $\operatorname{sgn}(\delta) \in R^{n}$ is a switching function vector, whose common element is a switching function in scalar case:

$$
\operatorname{sgn}_{i}\left(\delta_{i}\right)= \begin{cases}1 & \delta_{i}>0 \\ 0 & \delta_{i}=0 \\ -1 & \delta_{i}<0\end{cases}
$$

where $\delta_{i} \in \delta(i=1,2, \ldots, n)$.

The block diagram of the control scheme is shown in Figure 2.

Substituting (13), (15), (21) (22), (31), and (33) into (6), we obtain the following error dynamics:

$$
\dot{S}=A S+B,
$$

where $B=\left[\begin{array}{ll}0^{\mathrm{T}} & b^{\mathrm{T}}\end{array}\right]^{\mathrm{T}}$ and

$$
b=-\widetilde{\theta}^{1 \mathrm{~T}} F^{1}(q, \dot{q})-\widetilde{\theta}^{2 \mathrm{~T}} F^{2}(q, \ddot{q})+\varepsilon^{1}+\varepsilon^{2}+u_{s l}+d .
$$

The weight matrix updating algorithms are chosen as follows:

$$
\begin{aligned}
& \dot{\theta}^{1}=\eta_{1} F^{1}(q, \dot{q}) \delta^{\mathrm{T}}, \\
& \dot{\theta}^{2}=\eta_{2} F^{2}(q, \ddot{q}) \delta^{\mathrm{T}},
\end{aligned}
$$

where $\eta_{1}$ and $\eta_{2}$ are positive constants. Considering (24), there are always

$$
\begin{aligned}
& \dot{\tilde{\theta}}^{1}=\dot{\theta}^{1}, \\
& \dot{\tilde{\theta}}^{2}=\dot{\theta}^{2} .
\end{aligned}
$$

Theorem 4. Considering more concise expression (6) of system (1), if the controller is synthesized by (13), (15), (31), and (33) and the weight matrix is adjusted by adaptive mechanism (37), then the trajectory tracking error of system (6) converges to zero asymptotically.

Proof. Construct a Lyapunov function as follows:

$$
V=\frac{1}{2} S^{\mathrm{T}} P S+\frac{1}{2} \operatorname{tr}\left(\widetilde{\theta}^{1 \mathrm{~T}} \eta_{1}^{-1} \widetilde{\theta}^{1}\right)+\frac{1}{2} \operatorname{tr}\left(\widetilde{\theta}^{2 \mathrm{~T}} \eta_{2}^{-1} \widetilde{\theta}^{2}\right),
$$

where $P$ is a solution of (12).

Differentiate $V$ with respect to the state trajectories of (35), under (32) and (36), yielding

$$
\begin{aligned}
\dot{V}= & \frac{1}{2} S^{\mathrm{T}}\left(P A+A^{\mathrm{T}} P\right) S+\frac{1}{2} B^{\mathrm{T}} P S+\frac{1}{2} S^{\mathrm{T}} P B \\
& +\operatorname{tr}\left(\widetilde{\theta}^{1 \mathrm{~T}} \eta_{1}^{-1} \dot{\tilde{\theta}}^{1}\right)+\operatorname{tr}\left(\widetilde{\theta}^{2 \mathrm{~T}} \eta_{2}^{-1} \dot{\tilde{\theta}}^{2}\right) \\
= & -\frac{1}{2} S^{\mathrm{T}} Q S+\frac{1}{2}\left[\begin{array}{ll}
0^{\mathrm{T}} & b^{\mathrm{T}}
\end{array}\right] P S+\frac{1}{2} S^{\mathrm{T}} P\left[\begin{array}{l}
0 \\
b
\end{array}\right]
\end{aligned}
$$




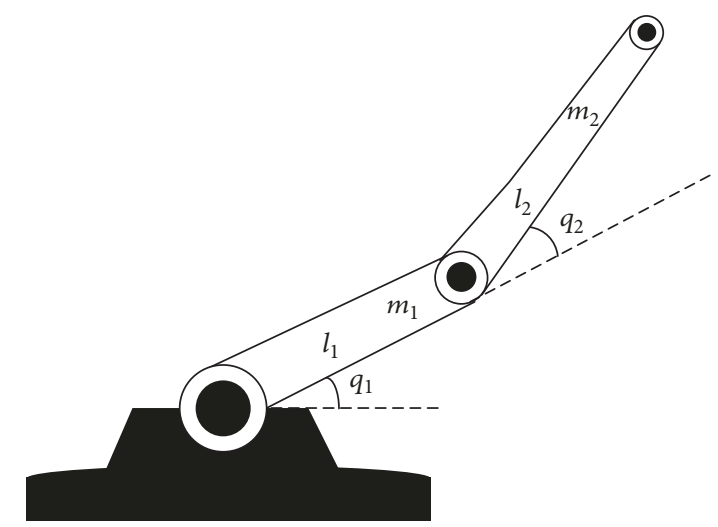

Figure 3: Two-link robotic manipulator.

$$
\begin{aligned}
& +\operatorname{tr}\left(\widetilde{\theta}^{1 \mathrm{~T}} \eta_{1}^{-1} \dot{\tilde{\theta}}^{1}\right)+\operatorname{tr}\left(\widetilde{\theta}^{2 \mathrm{~T}} \eta_{2}^{-1} \dot{\tilde{\dot{\theta}}}^{2}\right) \\
= & -\frac{1}{2} S^{\mathrm{T}} Q S+\frac{1}{2} b^{\mathrm{T}}\left[\begin{array}{ll}
P_{21} & P_{22}
\end{array}\right] S+\frac{1}{2} S^{\mathrm{T}}\left[\begin{array}{c}
P_{21} \\
P_{22}
\end{array}\right] b \\
& +\operatorname{tr}\left(\widetilde{\theta}^{1 \mathrm{~T}} \eta_{1}^{-1} \dot{\tilde{\theta}}^{1}\right)+\operatorname{tr}\left(\widetilde{\theta}^{2 \mathrm{~T}} \eta_{2}^{-1} \dot{\tilde{\dot{\theta}}}^{2}\right) \\
= & -\frac{1}{2} S^{\mathrm{T}} Q S+b^{\mathrm{T}} \delta+\operatorname{tr}\left(\widetilde{\theta}^{1 \mathrm{~T}} \eta_{1}^{-1} \dot{\tilde{\theta}}^{1}\right) \\
& +\operatorname{tr}\left(\widetilde{\theta}^{2 \mathrm{~T}} \eta_{2}^{-1} \dot{\tilde{\dot{\theta}}}^{2}\right) \\
= & -\frac{1}{2} S^{\mathrm{T}} Q S-F^{1 \mathrm{~T}}(q, \dot{q}) \widetilde{\theta}^{1} \delta-F^{2 \mathrm{~T}}(q, \ddot{q}) \widetilde{\theta}^{2} \delta \\
& +\left(\varepsilon^{1}+\varepsilon^{2}+u_{s l}+d\right)^{\mathrm{T}} \delta+\operatorname{tr}\left(\widetilde{\theta}^{1 \mathrm{~T}} \eta_{1}^{-1} \dot{\tilde{\theta}}^{1}\right) \\
& +\operatorname{tr}\left(\widetilde{\theta}^{2 \mathrm{~T}} \eta_{2}^{-1} \dot{\tilde{\theta}}^{2}\right) .
\end{aligned}
$$

Further, substitute the adaptive updating rules (37) and (38) into the above expression, and use Lemma 3, obtaining

$$
\dot{V}=-\frac{1}{2} S^{\mathrm{T}} Q S+\left(\varepsilon^{1}+\varepsilon^{2}+u_{s l}+d\right)^{\mathrm{T}} \delta .
$$

Then, substitute (31) and (33) into $\dot{V}$, displaying

$$
\begin{aligned}
\dot{V}= & -\frac{1}{2} S^{\mathrm{T}} Q S \\
& +\left(\varepsilon^{1}+\varepsilon^{2}-\left(\sigma^{1}+\sigma^{2}+d_{0}\right) \operatorname{sgn}(\delta)+d\right)^{\mathrm{T}} \delta .
\end{aligned}
$$

Considering (34), no matter $\delta_{i}>0, \delta_{i}=0$ or $\delta_{i}<0$, there are always

$$
\dot{V} \leq-\frac{1}{2} S^{\mathrm{T}} Q S \leq 0
$$

In fact, take (23) and Remark 2 into consideration; when $\delta_{i} \geq$ 0 , the latter part of (42) satisfies

$$
\begin{gathered}
\left(\varepsilon_{i}^{1}+\varepsilon_{i}^{2}-\left(\sigma^{1}+\sigma^{2}+d_{0}\right) \operatorname{sgn}\left(\delta_{i}\right)+d_{i}\right) \delta_{i} \\
=\left(\varepsilon_{i}^{1}+\varepsilon_{i}^{2}-\left(\sigma^{1}+\sigma^{2}+d_{0}\right)+d_{i}\right) \delta_{i} \leq 0 .
\end{gathered}
$$

And when $\delta_{i}<0$, the latter part of (42) satisfies

$$
\begin{gathered}
\left(\varepsilon_{i}^{1}+\varepsilon_{i}^{2}-\left(\sigma^{1}+\sigma^{2}+d_{0}\right) \operatorname{sgn}\left(\delta_{i}\right)+d_{i}\right) \delta_{i} \\
=\left(\varepsilon_{i}^{1}+\varepsilon_{i}^{2}+\left(\sigma^{1}+\sigma^{2}+d_{0}\right)+d_{i}\right) \delta_{i} \leq 0 .
\end{gathered}
$$

Now, we have proved that the Lyapunov function $V$ decreases monotonically. Thus, it can be concluded that the close loop system is globally stable and $S, \widetilde{\theta}^{1}, \widetilde{\theta}^{2}$ are uniformly bounded.

Furthermore, it is easy to show that $\theta^{1}$ and $\theta^{2}$ are uniformly bounded too. With boundedness of $f(q, \dot{q}, \ddot{q})$, we can say that the estimations $\widehat{f}^{1}\left(q, \dot{q} \mid \theta^{1}\right)$ and $\widehat{f}^{2}\left(q, \ddot{q} \mid \theta^{2}\right)$ are also bounded. Thus, it can be concluded that $u_{a l}$ in (13) and $u_{s l}$ in (31) are uniformly bounded. That is to say, all the right parts of (36) are bounded, and hence $b$ is bounded. Therefore, (35) implies that $\dot{S}$ is uniformly bounded, which tells us that $S$ will be uniformly continuous.

Let $V_{1}=V-\int_{0}^{t}\left(\dot{V}+(1 / 2) S^{\mathrm{T}} Q S\right) d \tau$. Because of the existence of $\dot{V} \leq-1 / 2 S^{\mathrm{T}} \mathrm{Q} S$, then $V_{1}(t) \geq 0$, so we can say that $V_{1}(t)$ has below boundedness. Further, as $\dot{V}_{1}=$ $-1 / 2 S^{\mathrm{T}} Q S$, then $\dot{V}_{1} \leq 0$; that is, $\dot{V}_{1}$ is semi-negativedefinite. Finally, the uniform continuousness of $S$ will make $\dot{V}_{1}$ uniformly continuous too. Hence, using Barbalat's lemma, we can deduce that $\lim V_{1}=0$; that is, when $t \rightarrow \infty$, we have $S \rightarrow 0$. Then the tracking error $e$ and its derivative $\dot{e}$ converge to zero asymptotically.

\section{Simulations Results}

In this section, we will take a two-link manipulator to verify the feasibility of the suggested controller. The configuration of the manipulator and its parameters are shown in Figure 3 [27-29]. 
The symbols in (1) are described as follows:

$$
\begin{aligned}
M(q) & =\left[\begin{array}{ll}
M_{11} & M_{12} \\
M_{21} & M_{22}
\end{array}\right], \\
h(q, \dot{q}) & =\left[\begin{array}{l}
h_{1} \\
h_{2}
\end{array}\right],
\end{aligned}
$$

where $h_{1}=-m_{2} l_{1} l_{2} \sin \left(q_{2}\right) \dot{q}_{2}\left(\dot{q}_{1}+\dot{q}_{2}\right)+\left(m_{1}+m_{2}\right) \cos \left(q_{2}\right) g$, $h_{2}=m_{2} l_{1} l_{2} \sin \left(q_{2}\right) \dot{q}_{1}^{2}, M_{11}=\left(m_{1}+m_{2}\right) l_{1}^{2}, M_{22}=m_{2} l_{2}^{2}$, and $M_{12}=M_{21}=m_{2} l_{1} l_{2} \cos \left(q_{1}-q_{2}\right)$.

The values of these body parameters are simply chosen as $m_{1}=m_{2}=1(\mathrm{~kg})$ and $l_{1}=l_{2}=1(\mathrm{~m})$. The initial angles are chosen as $q_{1}(0)=q_{2}(0)=0$, and their derivatives are $\dot{q}_{1}(0)=$ $\dot{q}_{2}(0)=0$. The desired reference trajectories are selected as $q_{d 1}=\sin t$ and $q_{d 2}=2 \sin t$. Let the outside disturbance be $\tau_{d}=\left[\begin{array}{ll}0.5 \cos (t) & 0.5 \sin (t)\end{array}\right]^{\mathrm{T}}$. Other parameters are $\alpha=$ $\beta=100 I_{2}, \sigma^{1}=\sigma^{2}=1, \eta=0.1, d_{0}=0.5$, and $Q=I_{4}$.

In simulations, we choose five fuzzy levels, that is, $\mathrm{NB}$, NS, ZO, PS, and PB on the universe of each input variable and we use the following Gaussian membership function [24]:

$$
\mu_{A_{i}^{l}}\left(x_{i}\right)=\exp \left[-\left(\frac{x_{i}-\bar{x}_{i}^{l}}{\pi / 24}\right)^{2}\right],
$$

where $\bar{x}_{i}^{l}$ are $-\pi / 6,-\pi / 12,0, \pi / 12$, and $\pi / 6$, respectively.

When the modeling uncertainties $\Delta M(q)$ and $\Delta h(q, \dot{q})$ change by $20 \%$, the corresponding simulation results are shown in Figures 4(a) and 4(b). From these simulation results, we can see that the designed controller guarantees actual trajectory tracking of the desired one completely. Meanwhile, when the uncertainties $\Delta M(q)$ and $\Delta h(q, \dot{q})$ change in a greater range, for example $50 \%$, the corresponding simulation results are listed in Figures 4(c) and 4(d). Figure 4(c) tells us that in this case there are tracking trajectory errors. However, if we make parameters $\alpha$ and $\beta$ larger, for example, let $\alpha=\beta=500 I_{2}$, then there are no tracking trajectory errors again. This is shown in Figures 4(e) and 4(f), while Figure 4(f) indicates that when these uncertainties change in a bigger width and parameters $\alpha, \beta$ are larger, the control inputs tend to be more bad.

In order to investigate the performance of the proposed controllers, we will give a simulated comparison of the robust control scheme $[23,30]$ in the end of this chapter. In this scheme, (4) becomes $\tau=h_{0}(q, \dot{q})+M_{0}(q) u+M_{0}\left(\ddot{q}_{d}-\alpha \dot{e}-\beta e\right)$ and the controller $u=-B^{\mathrm{T}} P S \rho^{2} /\left(\left|S^{\mathrm{T}} B P\right| \rho+\gamma\right)$, where $B$ is selected as $B=[0,0 ; 0,0 ; 1,0 ; 0,1]$, that is, the same as $[23,30], P$ is a solution of the Lyapunov equation (12), $\gamma$ is a very small positive number chosen as $\gamma=0.001$, and $\rho$ describes the upper bound of modeling uncertainties $\Delta M(q)$ and $\Delta h(q, \dot{q})$, which can be chosen with a greater value than the upper bound of the modeling uncertainties, such as $\rho=$ 10. All of the other used expressions and parameters do not change.
The simulation results adopting robust control scheme are shown in Figure 5, where Figures 5(a) and 5(b) are obtained when the modeling uncertainties $\Delta M(q)$ and $\Delta h(q, \dot{q})$ change by $20 \%$, Figures $5(\mathrm{c})$ and $5(\mathrm{~d})$ are obtained when the modeling uncertainties $\Delta M(q)$ and $\Delta h(q, \dot{q})$ change by $50 \%$, and Figures $5(\mathrm{e})$ and $5(\mathrm{f})$ are also obtained when the modeling uncertainties change by $50 \%$, but in the case of $\alpha=\beta=200 I_{2}$ rather than $\alpha=\beta=100 I_{2}$ which are used in Figures 5(a), 5(b), 5(c), and 5(d).

From these simulations results, we can see that the proposed controller in this paper is very effective and shows higher precision. In fact, $[23,30]$ tell us that the robust controller $u=-B^{\mathrm{T}} P S \rho^{2} /\left(\left|S^{\mathrm{T}} B P\right| \rho+\gamma\right)$ can only guarantee global uniform ultimate boundedness rather than global asymptotic stability.

\section{Conclusion}

This paper proposes an adaptive fuzzy control scheme for uncertain robotic system. Although the control scheme is proposed for uncertain robotic system, it can be suitable for a kind of MIMO system. First, we prove our control scheme effectiveness based on Lyapunov method. Then, we take a two-link manipulator to verify the feasibility of the suggested controller. The simulation results tells us that what we do is very meaningful.

However, it should be noted that the variable structure term $\operatorname{sgn}(\delta)$ is adopted to increase the robust performance, but it will generate "chattering" phenomenon, which may irritate unmolded high-frequency dynamics and even destroy the physical device. In order to weaken or eliminate the "chattering," there are some good directions stated as follows.

(1) The saturation function method, which is described below, can be used when frequency is too high, but using the saturation may degrade the robustness. It is very important to choose an appropriate boundary layer thickness $\mu$.

$$
\operatorname{sat}(\delta)= \begin{cases}\operatorname{sgn}\left(\frac{\delta}{\mu}\right), & \left|\frac{\delta}{\mu}\right| \geq 1 \\ \frac{\delta}{\mu} & \left|\frac{\delta}{\mu}\right|<1 .\end{cases}
$$

(2) Adjust the term gain switching online, study a new sliding mode control method, and so on.

\section{Conflicts of Interest}

The authors declare that they have no conflicts of interest.

\section{Acknowledgments}

This work is supported by the National Natural Science Foundation of China (11371221), the Natural Science Foundation of Shandong Province of China (ZR2014AM032), and the Project of Shandong Province Higher Educational Science and Technology Program (J16LI15). 


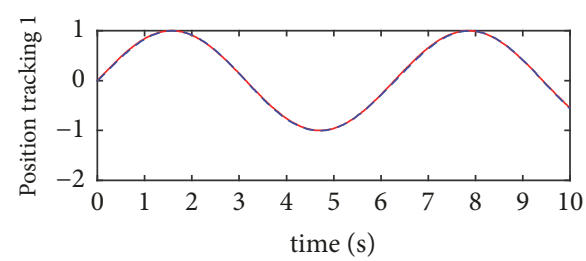

$-q d 1$

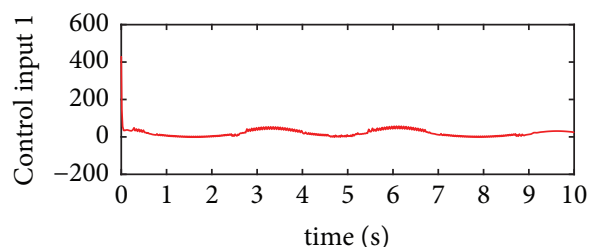

-- $q 1$
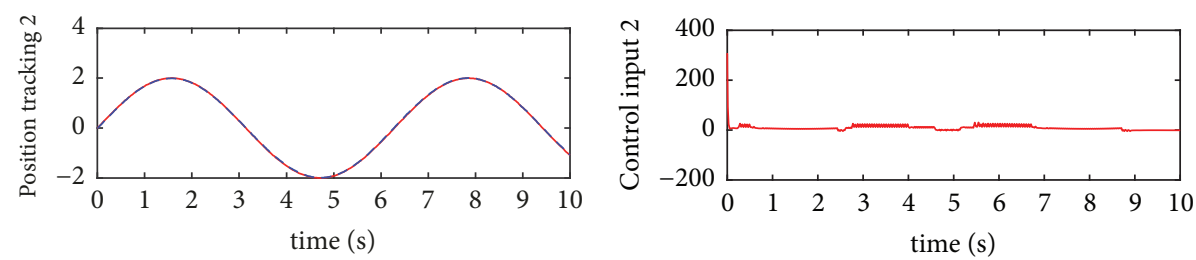

$-q d 2$
$--q^{2}$

(a) Tracking trajectory

(b) Control input

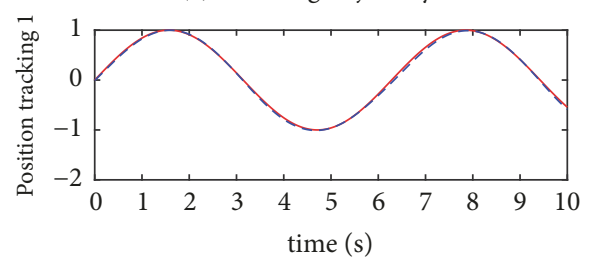

$\begin{array}{ll}- & q d 1 \\ -- & q 1\end{array}$
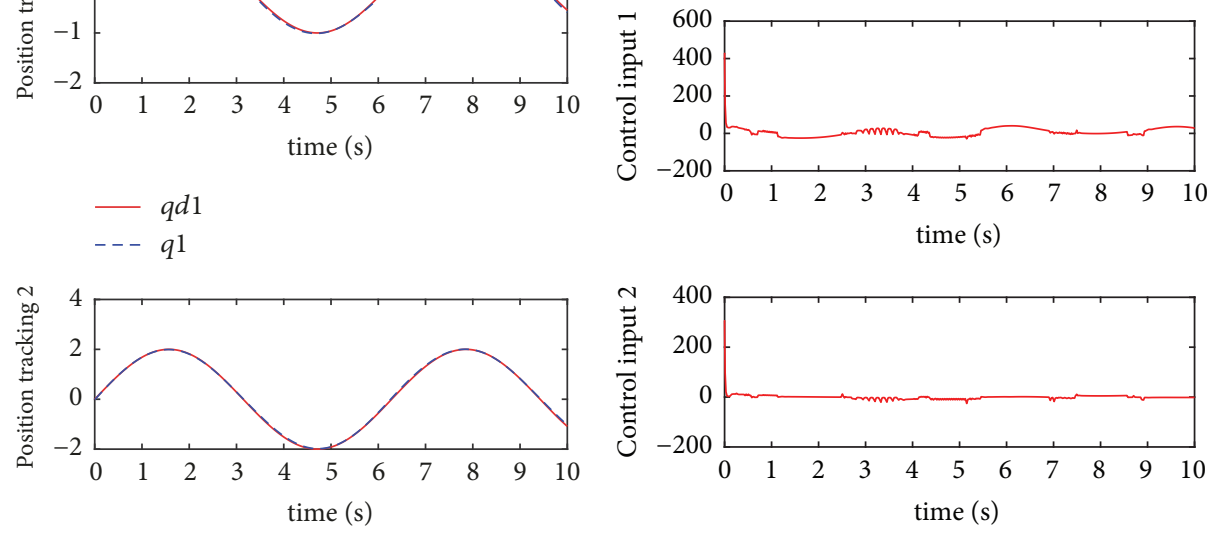

$-q d 2$
$---q^{2}$

(c) Tracking trajectory

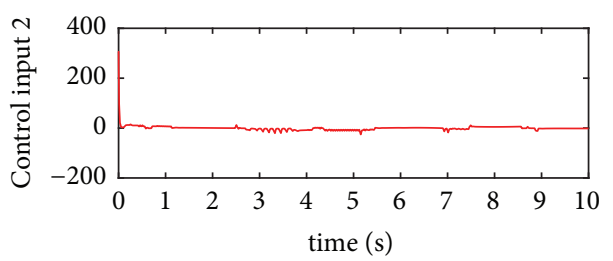

(d) Control input

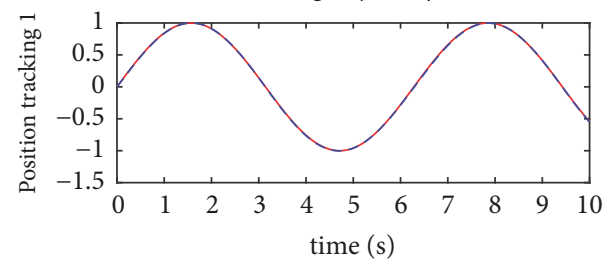

$-q d 1$

- - $q 1$
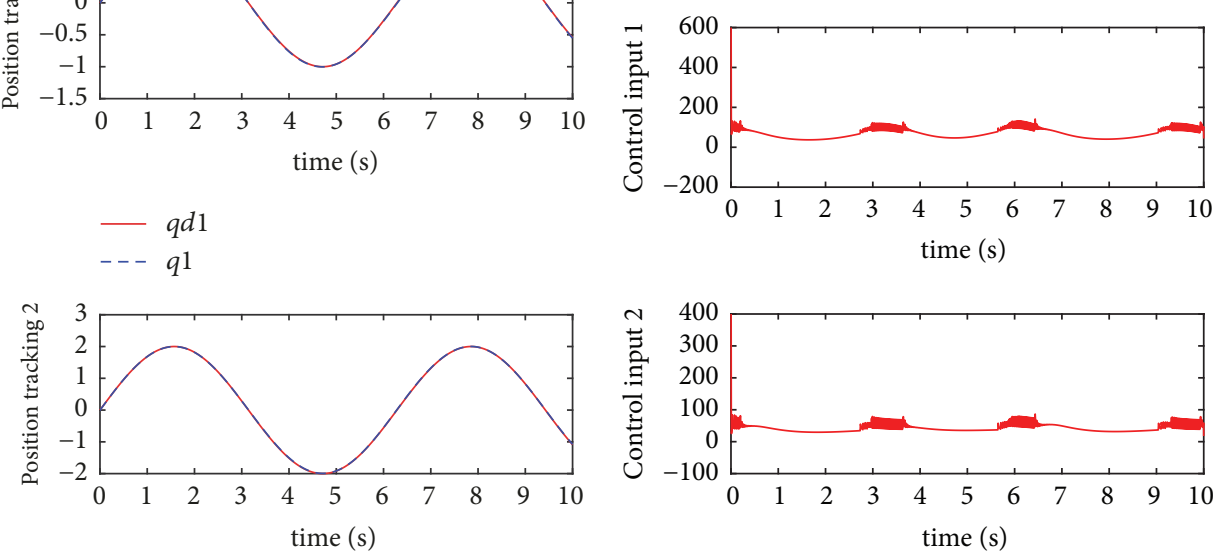

$\begin{array}{ll}- & q d 2 \\ --- & q 2\end{array}$

(e) Tracking trajectory

(f) Control input

FIGURE 4: Simulation results with proposed controller. 


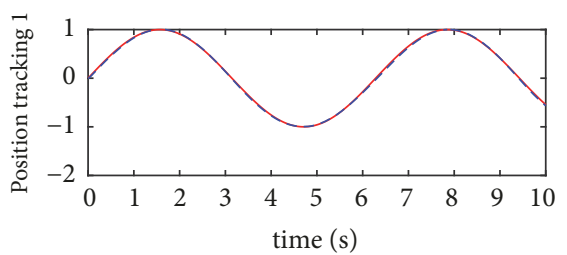

$-q d 1$

$$
---q 1
$$

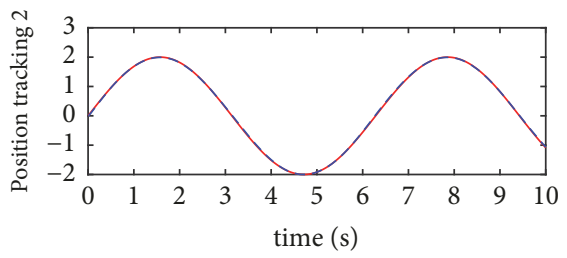

$$
\begin{aligned}
& -q d 2 \\
& ---q 2
\end{aligned}
$$

(a) Tracking trajectory

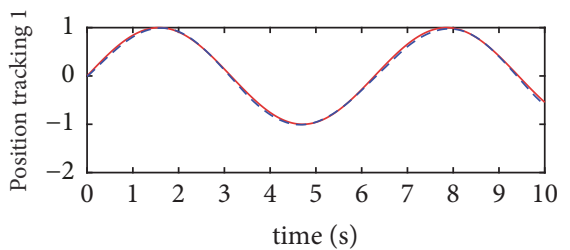

$$
q d 1
$$

$$
---q 1
$$

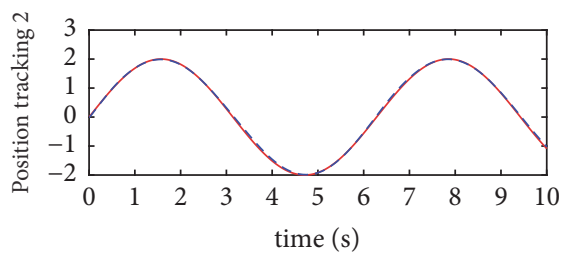

$$
\begin{aligned}
& -q d 2 \\
& --q^{2}
\end{aligned}
$$

(c) Tracking trajectory

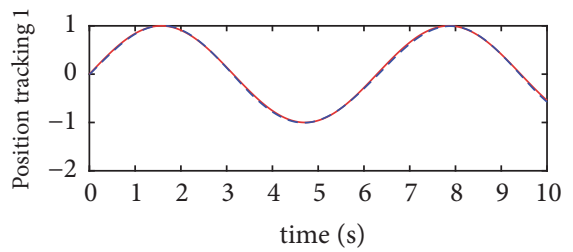

$q d 1$

$$
--q 1
$$

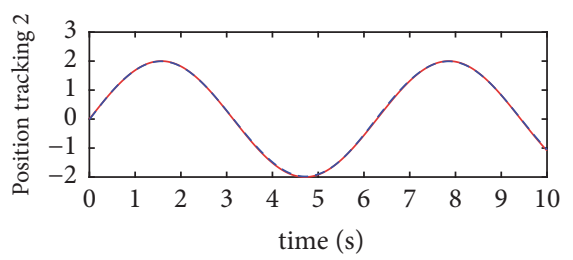

$$
\begin{aligned}
& -q d 2 \\
& ---q 2
\end{aligned}
$$

(e) Tracking trajectory

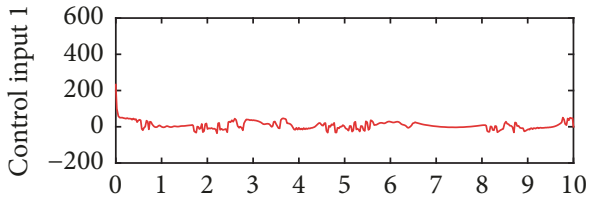

time (s)

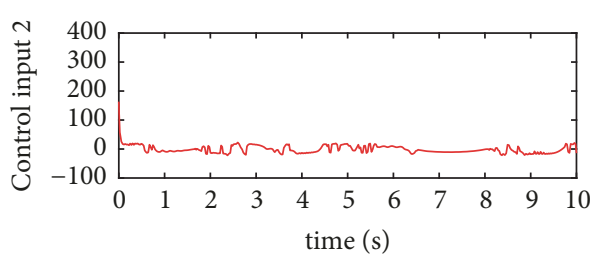

(b) Control input
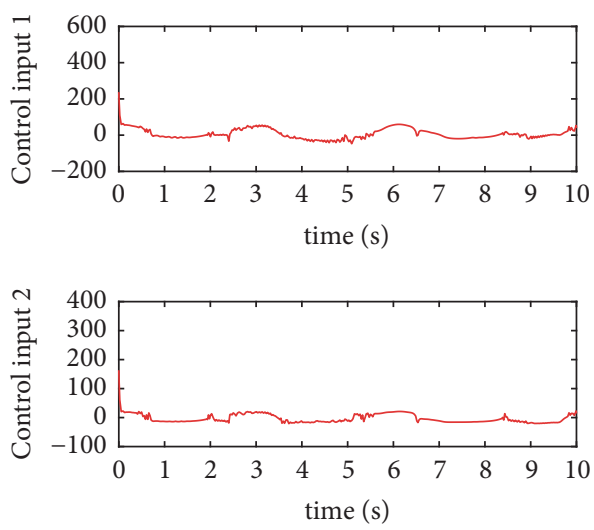

(d) Control input
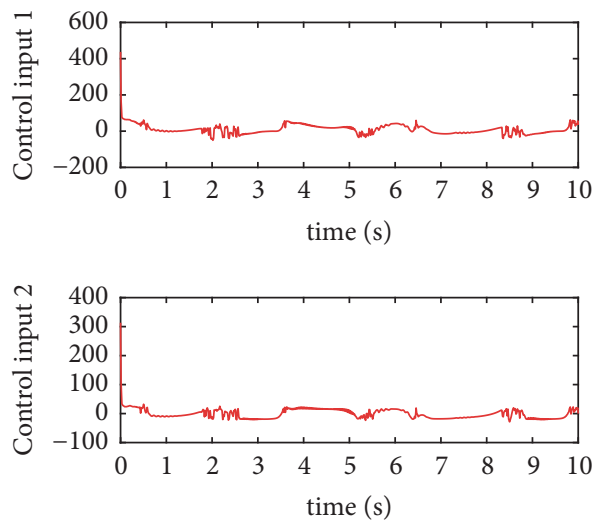

(f) Control input

FIGURE 5: Simulation results with robust controller. 


\section{References}

[1] L. Wang, T. Chai, and C. Yang, "Neural-network-based contouring control for robotic manipulators in operational space," IEEE Transactions on Control Systems Technology, vol. 20, no. 4, pp. 1073-1080, 2012.

[2] L. Yu, S. Fei, J. Huang, and Y. Gao, "Trajectory switching control of robotic manipulators based on RBF neural networks," Circuits, Systems and Signal Processing, vol. 33, no. 4, pp. 11191133, 2014.

[3] M. Kemmotsu and Y. Mutoh, "Control system of robot manipulator using linear time-varying controller design technique," in Proceedings of the 33rd Chinese Control Conference, pp. 28-30, Nanjing, China, 2014.

[4] T. Mai, Y. Wang, and T. Ngo, "Adaptive tracking control for robot manipulators using fuzzy wavelet neural networks," International Journal of Robotics and Automation, vol. 30, no. 1, pp. 26-39, 2015.

[5] K. Kherraz, M. Hamerlain, and N. Achour, "Robust neurofuzzy sliding mode controller for a flexible robot manipulator," International Journal of Robotics and Automation, vol. 30, no. 1, pp. 40-49, 2015.

[6] C. W. Tao, J. S. Taur, and M.-L. Chan, "Adaptive fuzzy terminal sliding mode controller for linear systems with mismatched time-varying uncertainties," IEEE Transactions on Systems, Man, and Cybernetics, Part B: Cybernetics, vol. 34, no. 1, pp. 255262, 2004.

[7] M. Chen, Q. Wu, C. Jiang, and B. Jiang, "Guaranteed transient performance based control with input saturation for near space vehicles," Science China Information Sciences, vol. 57, no. 5, pp. 1-12, 2014.

[8] A. Boulkroune, M. Tadjine, M. M'Saad, and M. Farza, "How to design a fuzzy adaptive controller based on observers for uncertain affine nonlinear systems," Fuzzy Sets and Systems, vol. 159, no. 8, pp. 926-948, 2008.

[9] V. Nekoukar and A. Erfanian, "Adaptive fuzzy terminal sliding mode control for a class of MIMO uncertain nonlinear systems," Fuzzy Sets and Systems, vol. 179, no. 1, pp. 34-49, 2011.

[10] Z. B. Du and S. S. Hu, "Fuzzy mixed $\mathrm{H}_{2} / \mathrm{H}_{\infty}$ sampled-data control for nonlinear systems," Control and Decision, vol. 32, no. 5, pp. 930-934, 2017.

[11] R. H. Du, Y. F. Wu, W. Chen, and Q. W. Chen, "Adaptive backstepping fuzzy control for servo systems with backlash," Control Theory \& Applications, vol. 30, no. 2, pp. 254-260, 2013 (Chinese).

[12] J. Fei and J. Zhou, "Robust adaptive control of MEMS triaxial gyroscope using fuzzy compensator," IEEE Transactions on Systems, Man, and Cybernetics, Part B: Cybernetics, vol. 42, no. 6, pp. 1599-1607, 2012.

[13] X. Bu, X. Wu, Z. Ma, and Y. Zhong, "Design of a modified arctangent-based tracking differentiator," Journal of Shanghai Jiaotong University, vol. 49, no. 2, pp. 164-168, 2015.

[14] C. Ham, Z. Qu, and R. Johnson, "Robust fuzzy control for robot manipulators," IEE Proceedings Control Theory and Applications, vol. 147, no. 2, pp. 212-216, 2000.

[15] Y. Q. Wei, J. D. Zhang, L. Hou, and Q. L. Chang, "Backstepping adaptive fuzzy control for two-link robot manipulators," vol. 10, pp. 303-308, 2013.

[16] M. M. Azimi and H. R. Koofigar, "Adaptive fuzzy backstepping controller design for uncertain underactuated robotic systems," Nonlinear Dynamics, vol. 79, no. 2, pp. 1457-1468, 2014.
[17] J. Fei and H. Ding, "Adaptive sliding mode control of dynamic system using RBF neural network," Nonlinear Dynamics, vol. 70, no. 2, pp. 1563-1573, 2012.

[18] J. Fei and C. Lu, "Adaptive sliding mode control of dynamic systems using double loop recurrent neural network structure," IEEE Transactions on Neural Networks and Learning Systems, vol. 29, no. 4, pp. 1275-1286, 2017.

[19] F. Lin, Robust Control Design: An Optimal Control Approach, John Wiley \& Sons, Chichester, UK, 2007.

[20] F. O. Rodriguez, J. J. Rubio, C. R. Gaspar, J. C. Tovar, and M. A. Armendariz, "Hierarchical fuzzy CMAC control for nonlinear systems," Neural Computing and Applications, vol. 23, no. 1, pp. 323-331, 2013.

[21] J. L. Zhou and W. H. Zhang, "Robust control for robots based on linear state equation," in Proceedings of the 3rd International Conference on Impulsive Dynamical Systems and Applications, pp. 454-459, Qingdao, China, 2006.

[22] Z. H. Man and M. Palaniswami, "Robust tracking control for rigid robotic manipulators," IEEE Transactions on Automatic Control, vol. 39, no. 1, pp. 154-159, 1994.

[23] J. L. Zhou and W. H. Zhang, "Robust tracking control for a kind of robot traces," Control Engineering of China, vol. 14, no. 3, pp. 336-339, 2007.

[24] B. K. Yoo and W. C. Ham, "Adaptive control of robot manipulator using fuzzy compensator," IEEE Transactions on Fuzzy Systems, vol. 8, no. 2, pp. 186-199, 2000.

[25] S. Islam and P. X. Liu, "Robust adaptive fuzzy output feedback control system for robot manipulators," IEEE/ASME Transactions on Mechatronics, vol. 16, no. 2, pp. 288-296, 2011.

[26] F. C. Sun, Z. Q. Sun, and G. Feng, "Design of adaptive sliding mode controller for robot manipulators," in Proceedings of the 5th IEEE Conference on Fuzzy Systems, pp. 817-823, New Orleans, La, USA, 1996.

[27] K.-K. D. Young, "Controller design for a manipulator using theory of variable structure systems," IEEE Transactions on Systems, Man, and Cybernetics, vol. 8, no. 2, pp. 101-109, 1978.

[28] P. V. Cuong and W. Y. Nan, "Adaptive trajectory tracking neural network control with robust compensator for robot manipulators," Neural Computing and Applications, vol. 27, no. 2, pp. 525-536, 2016.

[29] J. Zhou, "Adaptive fuzzy finite-time control for uncertain robotic manipulator," International Journal of Robotics and Automation, vol. 32, no. 2, pp. 134-141, 2017.

[30] Y. Dai, S. Shi, and N. Zheng, "Class of robust control strategies for robot manipulators with uncertainties," Acta Automatica Sinica, vol. 25, no. 2, pp. 204-209, 1999. 


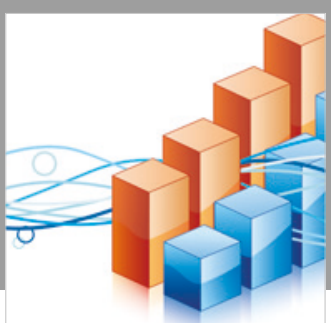

Advances in

Operations Research

\section{-n-m}
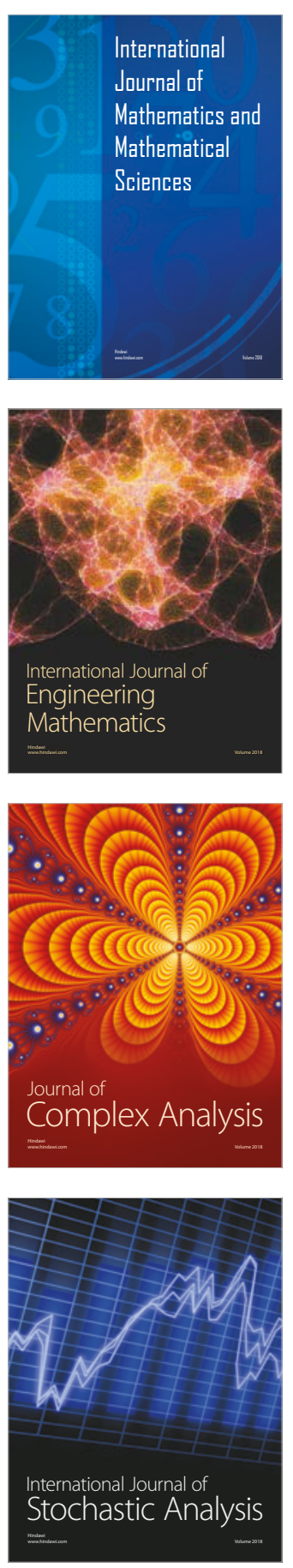
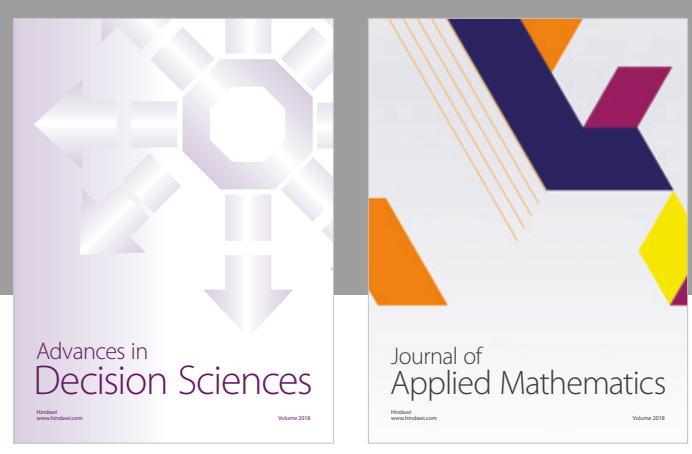

Journal of

Applied Mathematics
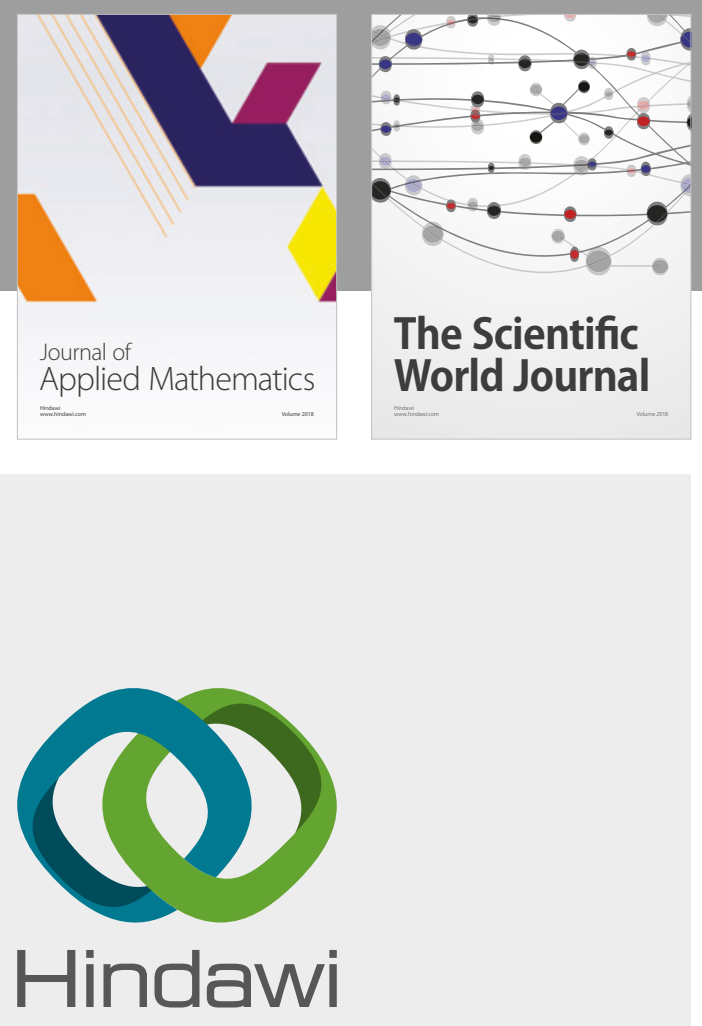

Submit your manuscripts at

www.hindawi.com

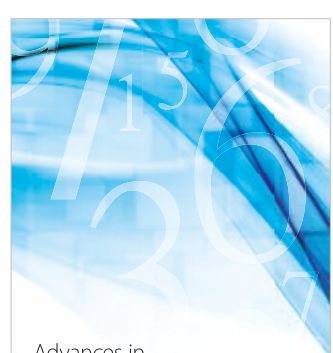

Advances in
Numerical Analysis
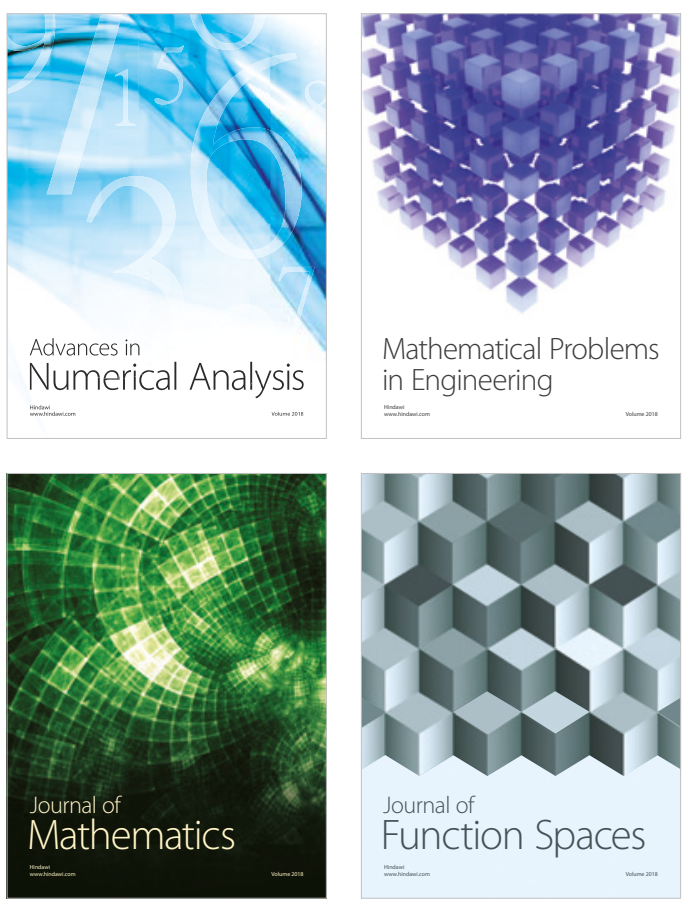

Mathematical Problems in Engineering

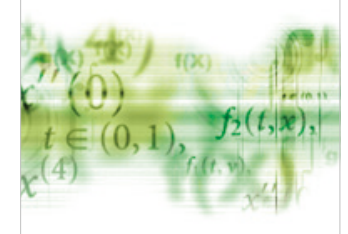

International Journal of

Differential Equations

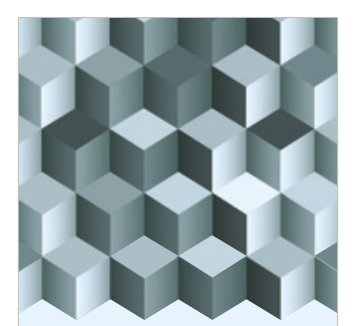

Journal of

Function Spaces

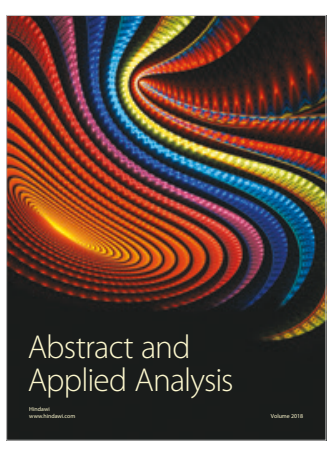

The Scientific

World Journal

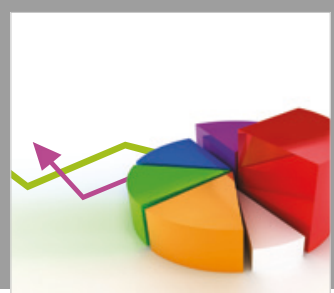

Journal of

Probability and Statistics
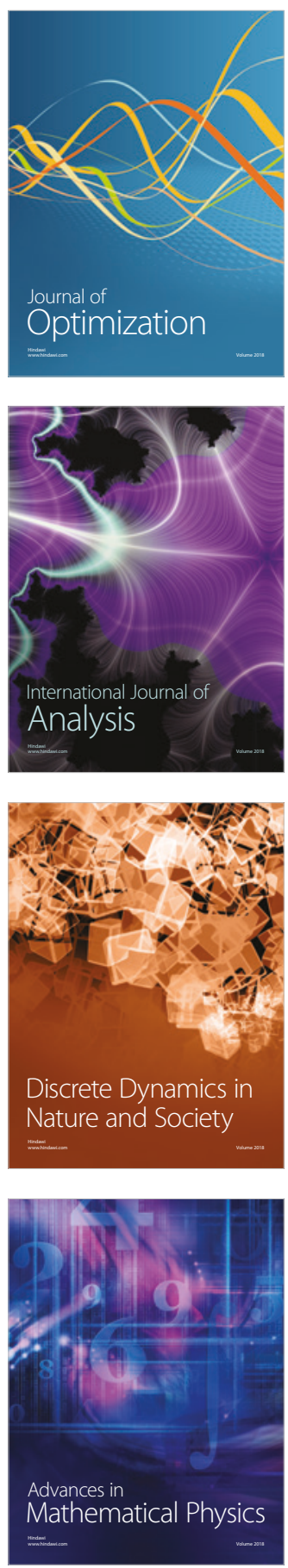